\title{
Structural properties of protective diamond-like-carbon thin films grown on multilayer graphene
}

\author{
Liu, Jian
}

2019-12-18

Liu , J , Muinos , H V , Nordlund, K \& Djurabekova , F 2019 , ' Structural properties of protective diamond-like-carbon thin films grown on multilayer graphene ' , Journal of Physics. Condensed Matter , vol. 31 , no. 50 , 505703 . https://doi.org/10.1088/1361-648X/ab4094

http://hdl.handle.net/10138/308762

https://doi.org/10.1088/1361-648X/ab4094

cc_by_nd

acceptedVersion

Downloaded from Helda, University of Helsinki institutional repository.

This is an electronic reprint of the original article.

This reprint may differ from the original in pagination and typographic detail.

Please cite the original version. 


\title{
Structural properties of protective diamond-like-carbon thin films grown on multilayer graphene
}

\author{
Jian Liu ${ }^{\mathrm{a}, \mathrm{b}}$, Henrique Vazquez Muinos ${ }^{\mathrm{a}}$, Kai Nordlund ${ }^{\mathrm{a}}$, Flyura \\ Djurabekova ${ }^{\mathrm{a}, *}$ \\ ${ }^{a}$ Helsinki Institute of Physics and Department of Physics, University of Helsinki, POB \\ 43, 00014, Helsinki, Finland \\ ${ }^{b}$ Department of Nuclear Science and Engineering, Nanjing University of Aeronautics and \\ Astronautics, Nanjing 210016, China
}

\begin{abstract}
In spite of the versatility of electronic properties of graphene, its fragility and low resistance to damage and external deformations reduce the practical value of this material for many applications. Coating of graphene with a thin layer of hard amorphous carbon is considered as a viable solution to protect the 2D material against accidental scratches and other external damaging impacts. In this study, we investigate the relationship between the deposition condition and quality of diamond-like-carbon (DLC) on top of multilayer graphene by means of molecular dynamics simulations. Deposition of carbon atoms with $70 \mathrm{eV}$ incident energy at $100 \mathrm{~K}$ resulted in the highest content of $s p^{3}$-bonded $\mathrm{C}$ atoms that amounted to $15.9 \%$. An increase of the number of dangling bonds at the interface between the top graphene layer and the DLC film indicates that increase of the incident energy reduces the adhesion quality of DLC thin film on graphene. Analysis of radial distribution function indicates that $s p^{3}$ hybridized carbon atoms tend to grow near already existing $s p^{3}$-atoms. This explains why the quality of the DLC structures grown on graphene have generally a lower content of $s p^{3} \mathrm{C}$ atoms compared to those grown directly on diamond. Ring analysis further shows that a DLC structure grown on the $s p^{2}$-rich structures like graphene contains a higher fraction of disordered ring structures.
\end{abstract}

Keywords: Diamond-like-carbon, Multilayer graphene, Molecular dynamics, Carbon atoms deposition

\footnotetext{
*e-mail: flyura.djurabekova@helsinki.fi
} 


\section{Introduction}

With the rapid development of nanoscience and nanotechnology, carbon nanomaterials, such as fullerenes [1], carbon nanotubes [2] and graphene [3], have gained much attention. Many scientific efforts are dedicated to study the properties and investigate potential applications of these carbon nanomaterials. Amongst these, graphene is the most promising stable $2 \mathrm{D}$ material. Moreover, its unique properties such as mechanical resistance [4], electrical [5] and thermal [6] conductivity etc, compared to conventional macro-materials make graphene a promising material in various applications such as gas sensing [7], water purification, [8] and touch screens [9] in the future. However, the attractive properties of graphene can alter severely due to mechanical wearing, scratching [10], or parasitic irradiation in space applications [11, 12]. The damage of graphene in daily used graphene-based appliances and equipments, will surely shorten the serving time of the latter. Thus, it is of great importance to find a compatible durable coating to protect graphene and enhance its resistant ability against external damage.

Diamond-like-carbon (DLC), is reported to be an ideal protective material for nanostructures and is able to enhance mechanical strength of nanomaterials [13-15]. DLC exhibits many desirable properties, such as ultra-high hardness and elastic moduli [16], low friction coefficient [17], transparency in the IR wavelength band [18] and chemical inertness [19]. Moreover, it is highly compatible with graphene structure. DLC coating is already widely used in many applications, e.g. as an antireflection layer for IR optic devices [20] or a protection layer for magnetic storage components [21] and an anticorrosion layer for biocompatible materials [22]. It will be utilized extensively even more in various fields like electronics, aviation, aerospace etc. [23-25]. In addition, some research has been carried out to analyze the protective properties of DLC films coating nanostructure both experimentally [15, 26-31] and by simulations [32-34]. In particular, Ren et al. [35] analyzed the effect of experimental conditions such as the combination of incident energy and ambient temperature on the quality of growing DLC film directly on diamond and on nanotubes placed on a diamond substrate, showing that a stable DLC can grow above a nanotube structure. The above-mentioned research shows that it is feasible to grow a DLC protective layer on top of carbon nanostructures. However, it is not entirely clear what defines the 
quality of the grown DLC films, in order to predict their protective properties. Unfortunately there are only few reports about the quality of DLC deposited on top of graphene in different conditions. DLC, which is a variety of amorphous carbon, shows diverse properties with a different ratio of $s p^{2}$ and $s p^{3}$ hybridization [36]. It was seen that the higher $s p^{3}$ fraction the DLC has, the harder and denser it becomes [37]. Therefore, in the current paper we aim to find out how the deposition condition during the growth of thin films can define the quality of DLC above graphene, in order to enable the growth of high quality DLC protective layers.

Atomistic simulation methods are powerful tools to study mechanisms of atomic migration and collision, and eventually, the formation of the bonds within the materials. In this paper, we utilized classical molecular dynamics (MD) to simulate and study the process of carbon ion beam deposition with different incident energies at different temperatures on the top of multilayer graphene. Then, we analyzed the atomic structure, density and $s p^{3}$ fraction of grown DLC films in different conditions in order to reveal what inherent mechanisms lead to failure of growth of stable and dense DLC structures.

\section{Simulation and analysis methods}

Simulations of DLC deposition process shown in this paper were carried out with classical MD code PARCAS [38, 39]. In order to describe the interaction between carbon atoms, the empirical analytical bond-order BrennerBeardmore potential [40-42] was applied. We used the extended low-end $(\mathrm{R}=1.95 \AA)$ and high-end $(\mathrm{S}=2.25 \AA)$ cutoff parameters, following Jäger and Albe's work [43] to improve the description of formation of $s p^{3}$-hybridization of carbon atoms. The extended cutoffs allowed depositing DLC with higher $s p^{3}$ fraction, which compares better to experimentally grown DLC films [35]. (COMMENT: Kai, could you write up this part with the potentials?) Unfortunately none of the classical potentials are able to predict correctly the formation of $s p^{3}$ bonded atoms [44], however, the selected potential with the extended cutoff can provide the understanding of comparative effect of DLC growth conditions. All the visualized simulation cells in this paper were obtained with the help of open visualization tool OVITO [45].

Figure 1 shows the schematic diagram of the initial multilayer graphene placed on top of the diamond substrate. For the sake of convenience, all $s p^{3}$ hybridized $\mathrm{C}$ atoms are colored red in this and the following figures, while $s p^{2}$-hybridized $\mathrm{C}$ atoms are colored blue. This structure was built up as 
follows. The diamond substrate with the $<111>$ surface normal and the size of $30 \times 30 \times 20$ Åincluded 3456 atoms. Six layers of graphene, 2304 atoms, were placed above the diamond substrate with the layer spacing of $3.35 \AA$ (the interlayer distance in graphite), and the distance between diamond surface and bottom graphene was also $3.35 \AA$. To minimize the energy of multilayer graphene system, all the six layers of graphene were stacked in the A-B Bernal order. Third, periodic boundary condition was set in lateral ( $x$ and $y$ ) directions, leaving $z$-direction an open surface to deposit DLC.

We used the Berendsen [46] thermostat to control the temperature at the lateral borders within the $5 \AA$ region and the two bottom layers of diamond atoms were fixed in order to dissipate heat and prevent the entire system from moving downwards during the continuous carbon ion beam irradiation. At last, incident carbon atoms were set $5 \AA$ above the top layer of graphene with the velocity directed perpendicular to the graphene layers in the direction of the surface. The incident energies for the deposition process was varied between 50,70, and $100 \mathrm{eV}$, in different sets of the subsequent deposition simulations. Before each carbon atom impact, a random cell shift in the $x y$ plane was performed to ensure that carbon atoms would hit the whole graphene layer uniformly. Each ion impact was simulated for the total time of $10 \mathrm{ps}$ to allow the generated heat to dissipate naturally at the boundaries of the simulation cell. As carbon atoms were arriving on graphene, the DLC film was gradually growing up and the initial incident position of depositing carbon atoms was also shifted up to ensure that there is always $5 \AA$ distance to the highest z-coordinate of an atom in the cell of the current size to avoid extreme repulsive forces caused due to inappropriately short distances between newly appeared incident atom and already existed DLC. The ambient temperature was set as $100 \mathrm{~K}$ during the DLC film deposition. For each energy, around 8000 carbon impacts were carried out to obtain the desired volume of DLC for further analysis.

To evaluate the quality of the grown DLC film, we analyzed the $s p^{3}$ fraction, mass density, radial distribution function (RDF), and primitive rings in all the grown structures. We set the first cutoff length as $1.9 \AA$, as an average value between the first and the second peaks in the total RDF, respectively, see Figure 3. All the atoms with the three neighbours within the selected cutoff were considered as three-fold carbon atoms, or as the $s p^{2}$ hybridized ones, while four-fold atoms are counted as having $s p^{3}$ hybridization. Apart from the total RDF for all atoms, we also computed the RDF for only the $s p^{2}$ and the $s p^{3}$ hybridized atoms to deconvolute the total RDF and ana- 


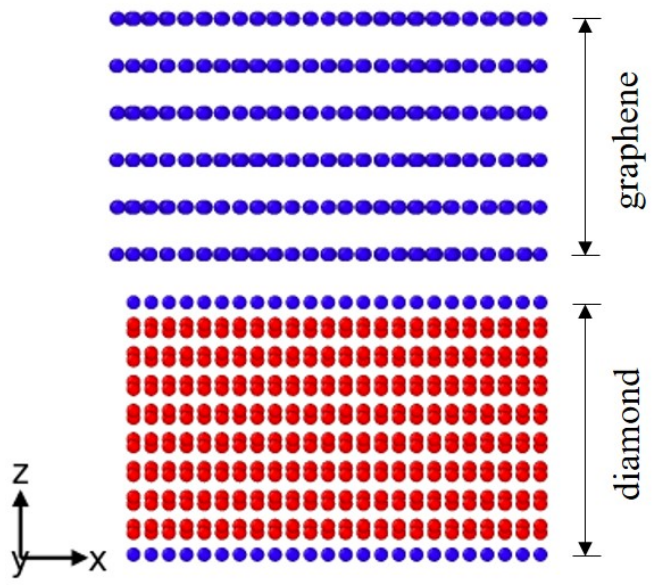

Figure 1: Schematic diagram of diamond and multilayer graphene model. Red atoms represent $s p^{3}$ hybridization while blue atoms stand for $s p^{2}$ hybridization.

lyze the short range order of both types of atoms in the grown DLC films. To inspect the total disorder degree, we also performed the primitive rings analysis [47]. In addition, the dangling bonds of carbon atoms located in the interface between graphene surface and bottom of the DLC were also analyzed to evaluate adhesion quality of the DLC film.

\section{Results and discussion}

\section{1. $s p^{3}$ fraction and mass density}

Figure 2 shows the distribution of the $s p^{3}$ fraction and mass density of the low-energy-ion deposited DLC film with the different incident energies along the $z$-axis (perpendicular to the surface) and corresponding simulation cells at $100 \mathrm{~K}$ and incident energies of 50,70 , and $100 \mathrm{eV}$, respectively. The four ticks to the right of the simulation cells are used to guide the eye to the location of the bottom and the top of the simulation cells, as well as of both interfaces (diamond-graphene and graphene-DLC). These four positions corresponds to four turning points in the relevant graphs, see Figure 2(a,c,e). In all these subfigures, the diamond and graphene regions are well visible since diamond has maximum density of $3.6 \mathrm{~g} / \mathrm{cm}^{3}$ and consists of pure $s p^{3}$ carbon atoms while graphene has minimum density of $1.8 \mathrm{~g} / \mathrm{cm}^{3}$ and is made of pure $s p^{2}$ carbon atoms. As $z$-coordinate increases, the average density increases fluctuating around $2.7 \mathrm{~g} / \mathrm{cm}^{3}$. At the same time, some increase in 
the $s p^{3}$ fraction is also observed. This behavior of the mass density and $s p^{3}$ fraction indicate the presence of the grown DLC film. We note here that the number of the $s p^{3}$-hybridized atoms is slightly higher in the middle of the cell, further away from the interface and the open surface, where excess of the free volume can impede formation of the $s p^{3}$-bonds. The stress exerted on $s p^{2}$ carbon atoms by deposition of energetic carbon atoms and the continuously growing DLC film, the $\pi \mathrm{C}-\mathrm{C}$ bond breaks converting into a $\sigma \mathrm{C}-\mathrm{C}$ bond. This process results in formation of four $\sigma$ covalent bonds corresponding to $s p^{3}$ hybridization instead of three $\sigma$ and one $\pi$ bonds corresponding to the $s p^{2}$ hybridization. On the other hand, the abundance of the $s p^{2}$-hybridized carbon atoms in graphene, the first layer of the growing film (right above graphene layers) consists mainly of the same $s p^{2}$ carbon atoms, constituting the loose amorphous carbon structure at the bottom of the DLC film.

While analyzing the $s p^{3}$ fractions in the DLC grown structures with different incident energies, we see that most $s p^{3}$ fraction forming in DLC films grown with $70 \mathrm{eV}$ incident energies than other two energies. This indicates that there exists an optimal incident energy to form highest $s p^{3}$ fraction. On the other hand, the images in Figure 2 show that with increase of incident energy of deposited $\mathrm{C}$ atoms, fewer layers of the graphene remain intact.

Reduction of the number of graphene layers means that graphene is partially consumed by the grown DLC film and some of the $s p^{2}$ carbon atoms from the original graphene layers transformed in the $s p^{3}$ hybridized atoms. Hence, the average $s p^{3}$ fraction obtained in the cell grown at $100 \mathrm{~K}$ with the $70 \mathrm{eV}$ incident carbon ions is higher than that of $50 \mathrm{eV}$. Although higher deposition energy increases the conversion of the activated graphene $s p^{2}$ atoms to become part of the DLC film, it also means that the $s p^{2}$-to- $s p^{3}$ conversion is promoted due to sufficient local stress generated by more energetic ions capable of penetrating deeper into the cell compared to those of $50 \mathrm{eV}$ energy. This effect makes a notable difference only at low temperature like $100 \mathrm{~K}$ since low temperature helps it stabilize the local concentration of the stress. Since $s p^{2}$ covalent C-C bonds are energetically more favorable [48], any temperature increase (also due to energetic ion impacts) in the disordered structure of amorphous carbon may reduce the local stress, causing spontaneous transition of forming $s p^{3} \mathrm{C}-\mathrm{C}$ bonds into $s p^{2} \mathrm{C}-\mathrm{C}$ bonds, reducing overall the $s p^{3}$ fraction.

The mass density of the DLC films grown under different conditions along with the corresponding $s p^{3}$ content are listed in Table 1 . We note that the simulation process of the film growth is time-consuming, this is why we report 

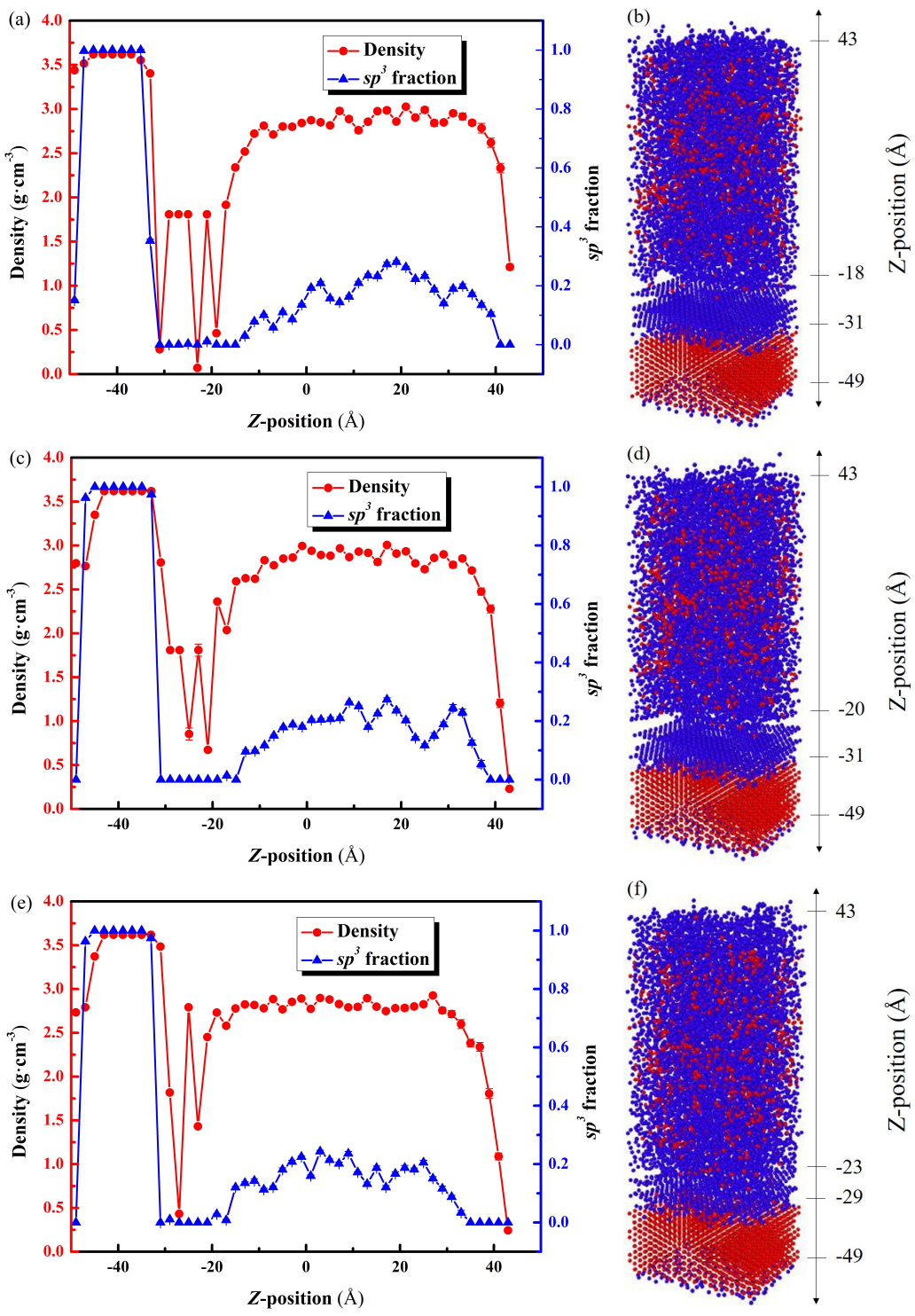

Figure 2: $s p^{3}$ fraction (right-hand $y$-axis) and mass density (left-hand $y$-axis) of the simulation cells as a function of $z$-coordinate with the incident energies of a): $50 \mathrm{eV} ; \mathrm{c}$ ): $70 \mathrm{eV}$; and f) $100 \mathrm{eV}$. The corresponding post-deposition simulation cells are shown in (b), (d), and (f), respectively. Red circles indicate the atoms with $s p^{3}$-hybridized bonds and blue are the atoms with $s p^{2}$ ones. The error bars show the standard deviation in the analysis of all the atoms in the corresponding structure. 
Table 1: Mass density $\left(\mathrm{g} / \mathrm{cm}^{3}\right)$ and $s p^{3}$ content (\%) of DLC films deposited at different incident energies.

\begin{tabular}{ccc}
\hline Energy $(\mathrm{eV})$ & $\begin{array}{c}\text { Mass density } \\
\left(\mathrm{g} / \mathrm{cm}^{3}\right)\end{array}$ & $s p^{3}$ content $(\%)$ \\
\hline 50 & 2.728 & 14.9 \\
70 & 2.766 & 15.9 \\
100 & 2.704 & 13.8 \\
\hline
\end{tabular}

here the results of analysis of a single structure for each energy, aiming mainly qualitative comparison of different deposition conditions. In Figure 2, where the error bars are the standard deviations from the mean value estimated either from the distribution or from the last frames of each of 10 last cascades (incident ion events). In this table, we see that the $s p^{3}$ fraction shows much stronger dependence on the incident energy than the average mass density of the grown DLC.

To investigate this effect further we plot now the radial distribution functions for two grown DLC structures with the highest and lowest content of $s p^{3}$ atoms to identify the difference in the short range order of all the carbon atoms within this structures. Figure 3 shows the radial distribution function grown with 70 and $100 \mathrm{eV}$, as well as deconvolution of the peak into the $s p^{2}$ and $s p^{3}$ components. We show all bonds within the high-end cutoff parameter $2.25 \AA$ of the Brenner potential, which includes only the first nearest neighbors. We see that the expected value of the bond length in the entire structure that was grown at $100 \mathrm{~K}$ is around $1.49 \pm 0.07 \AA$ and is independent of incident energy. Hereafter the error bars indicate the standard deviation in the value of the bond length for all the atoms in the corresponding structure. For comparison, the bond lengths in graphene and diamond structures in the Brenner interatomic potential with the extended cutoffs used in the present study are $1.459 \pm 0.048 \AA$ and $1.545 \pm 0.0001 \AA$, respectively. These values are close to those reported in the experiments, at least for the diamond structure it is known to be $1.54 \AA$ [49]. The bond length in graphene can be estimated as a weighted average between two single bonds and one double bond $\left(a_{C=C}=1.34 \AA[50]\right)$, which amounts to $1.47 \AA$.

When we analyze separately the expected values of the $s p^{2}$ - and $s p^{3}$-bond lengths, we found them to be the same in both structures shown in Figure 3 and amounted to $1.46 \pm 0.04 \AA$ and $1.6 \pm 0.09 \AA$, respectively. We see that the average bond length in the grown DLC structures for the $s p^{2}$-bonded 

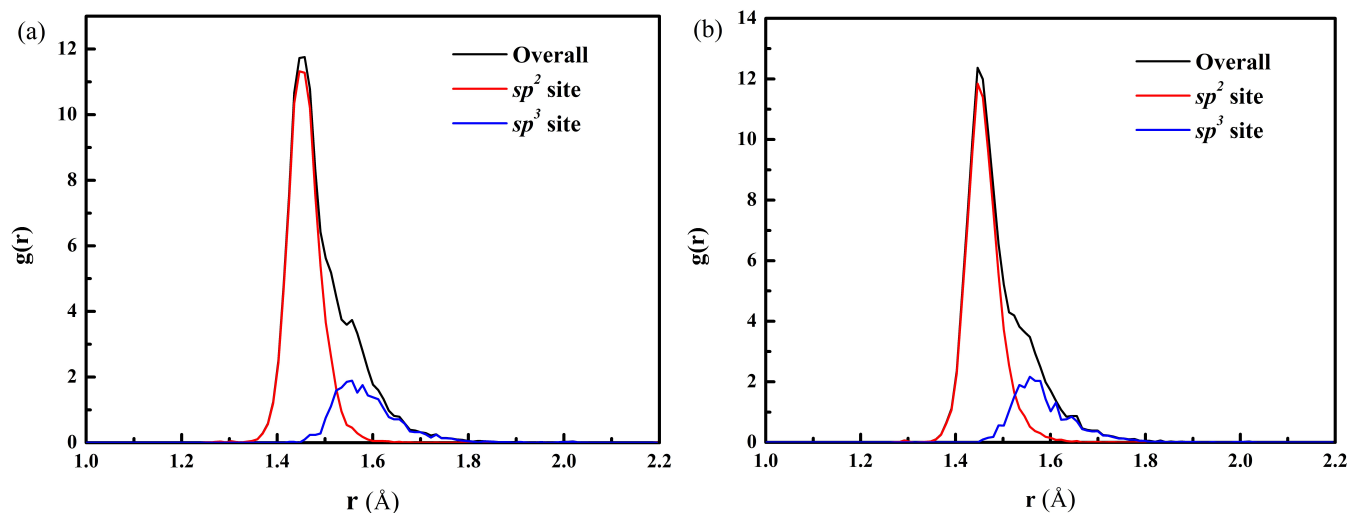

Figure 3: Radial distribution function $\mathrm{g}(\mathrm{r})$ for DLC deposited at $100 \mathrm{~K}$ under incident energies of (a) 70 and (b) $100 \mathrm{eV}$, respectively. The contributions of $s p^{2}$ and $s p^{3}$ coordination are presented separately in red and blue color.

atoms is very close to that in ideal graphene, while the $s p^{3}$-bonds are slightly stretched. Also there is some variation in the mean values of the $s p^{3}$-bond lengths because of relatively small number of the atoms with such bonds, the amount of these atoms clearly affects the average bond length in the entire structure.

Overall, in both cases we observe much higher peak of the $s p^{2}$-bonds compared to $s p^{3}$ ones as seen in Figure 3, even for the best structures with the highest density and highest $s p^{3}$-content. As it was shown in [35], $s p^{3}$ carbon atoms tend to grow near the atoms which already are $s p^{3}$-bonded as in diamond. Since we deposited the DLC film on top of graphene layers, the deposited atoms were landing next to the atoms with the $s p^{2}$-bonding, forming the same $s p^{2}$-bonds in more preferable manner. In other words, we observe the lack of formation of the $s p^{3}$-bonded carbon atoms during the initial stage of film deposition, which is consistent with the observations in [35].

Although the bond length of the $s p^{2}$-hybridized atoms is shorter, indeed they form only if there is sufficient free volume in the structure. It is also obvious in the values of the mass densities of the grown DLC structures: The higher the $s p^{3}$ content in the grown film, the higher its mass density is. In graphite, this inconsistency is explained by the large interlayer distance, while the interlayer distance in amorphous carbon is ill-defined. This is why we further analyze the average atomic volume in the DLC structures with the highest and the lowest $s p^{3}$ fraction. For this purpose we employ the 
Table 2: Average atomic volume with the standard deviation calculated for the carbon atoms in the DLC films deposited at different temperatures and incident energies.

\begin{tabular}{ccc}
\hline $\begin{array}{l}\text { Energy } \\
(\mathrm{eV})\end{array}$ & \multicolumn{2}{c}{ Average atomic volume $\left(\AA^{3}\right)$} \\
$s p^{2}$ atoms & $s p^{3}$ atoms \\
\hline 50 & $7.53 \pm 1.59$ & $6.08 \pm 0.65$ \\
70 & $7.44 \pm 1.12$ & $6.12 \pm 0.66$ \\
100 & $7.58 \pm 1.31$ & $6.15 \pm 0.61$ \\
\hline
\end{tabular}

Voronoi tessellation analysis available in OVITO software. According to this analysis, the average atomic volumes in multilayer graphene and diamond are $9.233 \pm 0.345 \AA^{3}$ and $5.678 \pm 0.001 \AA^{3}$, respectively.

In Table 2, we list the average atomic volume separately for $s p^{2}$ and $s p^{3}$ atoms in DLC films deposited at different incident energies at $100 \mathrm{~K}$. We see that the average atomic volume is larger in the cells with the lowest content of $s p^{3}$ bonding, which explains the lower mass density. It is surprising that the atomic volume for the $s p^{3}$-bonded atoms is very similar in all structures although it is slightly larger than in pure diamond. This indicates that the formed $s p^{3}$ atoms are stable and the bond stretching is mainly explained by the large amount of the $s p^{2}$-bonded atoms, which occupy much larger volume per atom. The slightly smaller atomic volume for the $s p^{2}$-bonded atoms are explained by densification during the impact.

\subsection{Ring analysis}

Different atomic volumes occupied by the $s p^{2}$-bonded atoms indicates that there can be structural differences in the grown films. To investigate the nature of this differences, we performed the analysis of the primitive rings for quantitative investigation of the degree of long range disorder in the deposited DLC films. We chose the same structures as analyzed in Figure 2, namely the films grown at $100 \mathrm{~K}$ with all three incident energies. For this analysis we used the method described in Ref. [47], excluding the top 20 $\AA$ of the DLC structure.

In Figure 4, we see that the three DLC structures grown at $100 \mathrm{~K}$ have similar distributions of the primitive rings. We see mainly the seven- to tenmember rings, although the number of the six-member rings is only slightly smaller than the larger rings. The five-member rings quite prominent in these structures as well. We see that the amount of the primitive rings with the large number members starts decreasing above 8-member rings in 


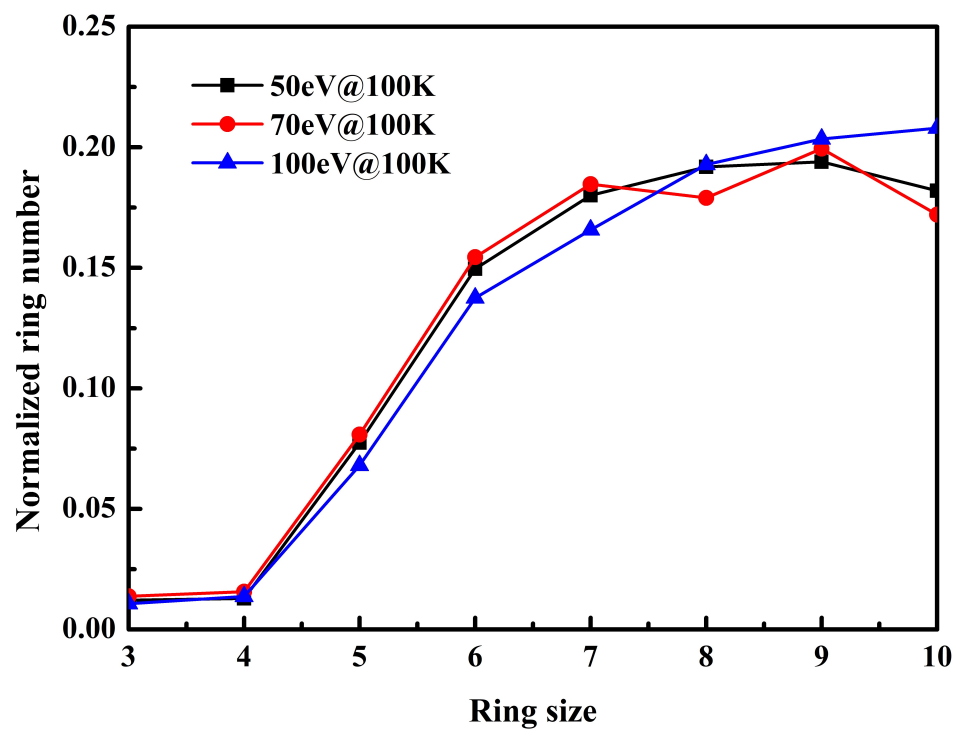

Figure 4: Normalized ring number as a function of ring size at $100 \mathrm{~K}$ under 50, 70, and $100 \mathrm{eV}$, respectively.

the more successful films with the larger quantity of the $s p^{3}$ atoms, while the film grown with the $100 \mathrm{eV}$ incident energy exhibits the presence of the primitive rings with the higher number of ten-member rings, which indicates the reduction of structural quality of the film. Compared to the structure of the disordered amorphous carbon rather than graphite indicating that they are quite disordered since the six-element ring represents the structure more close to diamond and is the prefect primitive ring structure. This could be attributed to the relatively low $s p^{3}$ fraction in Table 1 compared to Ref. [35]. Even so, DLC generated under $100 \mathrm{eV}$ shows more disordered structure than that under 50 and $70 \mathrm{eV}$ due to the relatively low fraction of six-element rings and high fraction of ten-element rings, meaning that $100 \mathrm{eV}$ produced poorer quality of DLC comparing to other two energies when considering previous data and analysis. DLC deposited under $70 \mathrm{eV}$ is only less disordered in a tiny degree than that under $50 \mathrm{eV}$ comparing the six- and other-element rings. Hence, considering the $s p^{3}$ fraction, mass density, and disorder degree, we can draw the conclusion that $70 \mathrm{eV}$ incident energy will lead to better quality of DLC deposited by carbon atoms continuously implanting. 


\subsection{Dangling bonds}

The quality of DLC film is defined not only by the high $s p^{3}$ fraction and mass density, but also by the strength of adhesion of the film to the graphene top layer. Loose adhesion of the film to the substrate may result in sliding of the film along the graphene surface under external force. Since the C$\mathrm{C}$ covalent bonds are strong and stable, the atoms with less neighbors than three at least will weaken the adhesion ability of the film in general. In Figure 5 we plot the number of atoms with dangling bonds $(<3$ neighbors) as a function of the $z$-coordinate. We also observe clear peaks at the positions corresponding to the interfaces between diamond and graphene, graphene and DLC, and at the open surface of DLC film. Unsaturated dangling bonds increase the energy of the interface reducing the barrier needed to detach the DLC film from the graphene surface.

Since we are interested in the interface between graphene and DLC, in this study we focus on the second peak from the left in Figure 5. Different symbols in the same plot show the results obtained at $100 \mathrm{~K}$ with different incident energies. In Figure 5, there are slightly less atoms with dangling bonds created by ions with $100 \mathrm{eV}$ than with $70 \mathrm{eV}$ and the largest number of the dangling bonds is observed for the lowest incident energy. This clearly indicates that the high incident energy decreases the number of dangling bonds due to more intensive displacement cascades and activated atoms, which helps energetic atoms at the interface form bonds with others. The analysis of the content of dangling bonds in the graphene-DLC interface shows a clear competition between the thermal and radiation-induced processes. In this study, ambient temperature $(100 \mathrm{~K})$ is quite low. Therefore, the thermal velocities are very small and the atoms do not have opportunity to saturate the dangling bonds without additional energy introduced by an impacting ion.

Based on this analysis, we conclude that the quality of film adhesion to multilayer graphene increases with increasing incident energy that counterbalanced by the poorer quality of the grown film structure at high energy. This is why, it is important to optimize the film growth condition by carefully selecting the energy of carbon ions during the deposition of the film. Since the peaks for the dangling bonds at this interface created by 70 and $100 \mathrm{eV}$ are almost the same, we conclude that the ions with $70 \mathrm{eV}$ transfer the optimal amount of energy to the lattice atoms to maximize the effect of bond saturation and protect more beneath graphene layers. At lower energy, the transferred energy may not be sufficient and at higher energy induces too 


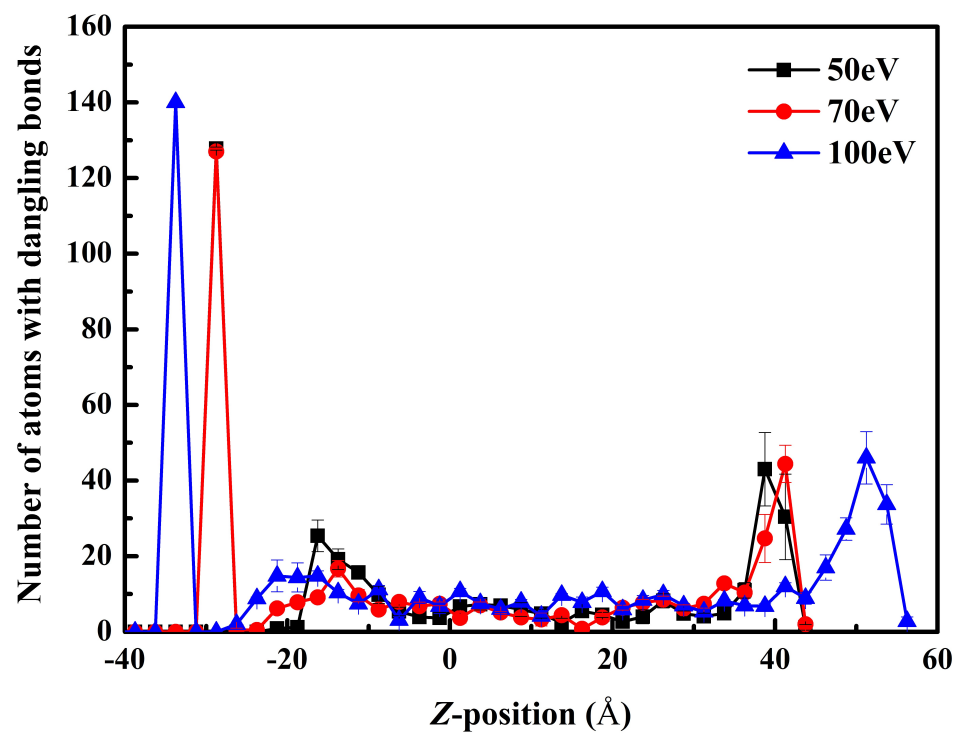

Figure 5: Number of atoms with dangling bonds as a function of z-position under different incident energies at $100 \mathrm{~K}$. Each data point is averaged on the last frame of last 10 incidents and with error bars representing standard deviation.

strong movement of atoms that the bonds remain dangling and also graphene layers are consumed (see the remaining intact graphene layers).

\section{Conclusions}

In this study, we analyzed the quality of the DLC films deposited on multilayer graphene as a protective layer with different energy of incident carbon ions. We showed that in spite of $s p^{2}$-bonding of carbon atoms in graphene, which impedes the formation of $s p^{3}$-hybridization, DLC film can grow above graphene by careful selection of optimal deposition condition. We saw that $s p^{3}$ hybridization tends to appear in the middle part of DLC along z-direction due to the pressure induced transformation from $s p^{2}$ to $s p^{3}$ hybridization. Deposition at $70 \mathrm{eV}$ resulted in most fraction of $s p^{3}$-bonded atoms and the highest mass density. They dropped with both increasing and decreasing incident energies. In the films, the $s p^{2}$-bond length was very close on average to that of graphene, while the $s p^{3}$-bond length was slightly longer than that in diamond. Clearly the atomic volume occupied by the $s p^{2}$ bonded atoms was much larger than that of $s p^{3}$-bonded atoms, but yet, much smaller than in multilayer graphene. By analyzing the primitive rings in the 
grown structures, we also concluded that the DLC grows above graphene is mainly disordered structure with a small amount of stable five and sixmember rings. At the same time, the quality of the film adhesion improved with increase of incident energy at $100 \mathrm{~K}$. The best adhesion was achieved with the energy of $100 \mathrm{eV}$. After comprehensive consideration of various situations, we concluded that $70 \mathrm{eV}$ incident energy can provide best quality of DLC film while it can also retain good adhesion ability with graphene layers.

\section{Acknowledgements}

The sponsorship of Open Fund of Key Laboratory of Materials Preparation and Protection for Harsh Environment (Nanjing University of Aeronautics and Astronautics), Ministry of Industry and Information Technology (No. 56XCA18159-2) is gratefully acknowledged. This work is also supported by the Fundamental Research Funds for the Central Universities (Grant No. NJ20150021 and No. NJ20170012), the Funding of Jiangsu Innovation Program for Graduate Education (Grant No. KYLX15_0306) and the Fundamental Research Funds for the Central Universities. The support provided by China Scholarship Council (CSC) during a visit of Jian Liu to University of Helsinki is acknowledged as well.

\section{References}

[1] H. Murayama, S. Tomonoh, J. M. Alford, M. E. Karpuk, Fullerene production in tons and more: from science to industry, Fuller. Nanotub. Car. N. 12 (2005) 1-9.

[2] R. H. Baughman, A. A. Zakhidov, W. A. De Heer, Carbon nanotubesthe route toward applications, Science 297 (2002) 787-792.

[3] H. Huang, X. Tang, F. Chen, J. Liu, H. Li, D. Chen, Graphene damage effects on radiation-resistance and configuration of copper-graphene nanocomposite under irradiation: A molecular dynamics study, Sci. Rep. 6 (2016) 39391.

[4] V. B. Mohan, H. Souri, K. Jayaraman, D. Bhattacharyya, Mechanical properties of thin films of graphene materials: A study on their structural quality and functionalities, Curr. Appl. Phys. 18 (2018) 879-885. 
[5] H. Sun, C. Fu, Y. Gao, P. Guo, C. Wang, W. Yang, Q. Wang, C. Zhang, J. Wang, J. Xu, Electrical property of macroscopic graphene composite fibers prepared by chemical vapor deposition, Nanotechnology 29 (2018) 305601.

[6] G. Qin, Z. Qin, H. Wang, M. Hu, On the diversity in the thermal transport properties of graphene: A first-principles-benchmark study testing different exchange-correlation functionals, Comp. Mater. Sci. 151 (2018) 153-159.

[7] F. A. de Souza, R. G. Amorim, J. Prasongkit, W. L. Scopel, R. H. Scheicher, A. R. Rocha, Topological line defects in graphene for applications in gas sensing, Carbon 129 (2018) 803-808.

[8] S. Dervin, D. D. Dionysiou, S. C. Pillai, 2d nanostructures for water purification: graphene and beyond, Nanoscale 8 (2016) 15115-15131.

[9] U. Khan, T.-H. Kim, H. Ryu, W. Seung, S.-W. Kim, Graphene tribotronics for electronic skin and touch screen applications, Adv. Mater. 29 (2017) 1603544.

[10] M.-S. Lee, K. Lee, S.-Y. Kim, H. Lee, J. Park, K.-H. Choi, H.-K. Kim, D.-G. Kim, D.-Y. Lee, S. Nam, et al., High-performance, transparent, and stretchable electrodes using graphene-metal nanowire hybrid structures, Nano Lett. 13 (2013) 2814-2821.

[11] X. Liu, J. Pu, L. Wang, Q. Xue, Novel dlc/ionic liquid/graphene nanocomposite coatings towards high-vacuum related space applications, Journal of Materials Chemistry A 1 (2013) 3797-3809.

[12] E. J. Siochi, Graphene in the sky and beyond, Nature nanotechnology 9 (2014) 745.

[13] J. Robertson, Properties of diamond-like carbon, Surface and Coatings Technology 50 (1992) 185-203.

[14] A. Voevodin, M. Donley, J. Zabinski, Pulsed laser deposition of diamond-like carbon wear protective coatings: a review, Surface and Coatings Technology 92 (1997) 42-49. 
[15] C. Wei, J.-F. Yang, F.-C. Tai, The stress reduction effect by interlayer deposition or film thickness for diamond like carbon on rough surface, Diam. Relat. Mater. 19 (2010) 518-524.

[16] Y. J. Won, H. Ki, Effect of film gradient profile on adhesion strength, residual stress and effective hardness of functionally graded diamond-like carbon films, Appl. Surf. Sci. 311 (2014) 775-779.

[17] K. Ankit, A. Varade, N. Reddy, S. Dhan, M. Chellamalai, P. Krishna, N. Balashanmugam, Synthesis of high hardness, low cof diamond-like carbon using rf-pecvd at room temperature and evaluating its structure using electron microscopy, Diam. Relat. Mater. 80 (2017) 108-112.

[18] N. Klyui, A. Liptuga, V. Lozinskii, A. Lukyanov, A. Oksanich, V. Terban, Application of diamond-like carbon films to increase transmission of semi-insulating gaas crystals in the ir spectral range, Tech. Phys. Lett. 38 (2012) 609-612.

[19] K. Bewilogua, D. Hofmann, History of diamond-like carbon films - from first experiments to worldwide applications, Surf. Coat. Tech. 242 (2014) $214-225$.

[20] K. Ankit, A. Varade, N. Reddy, S. Dhan, M. Chellamalai, N. Balashanmugam, P. Krishna, Synthesis of high hardness ir optical coating using diamond-like carbon by pecvd at room temperature, Diam. Relat. Mater. 78 (2017) 39-43.

[21] P. R. Goglia, J. Berkowitz, J. Hoehn, A. Xidis, L. Stover, Diamond-like carbon applications in high density hard disc recording heads, Diam. Relat. Mater. 10 (2001) 271-277.

[22] A. Grill, Diamond-like carbon coatings as biocompatible materials - an overview, Diam. Relat. Mater. 12 (2003) 166-170.

[23] L. Bai, G. Zhang, Z. Lu, Z. Wu, Y. Wang, L. Wang, P. Yan, Tribological mechanism of hydrogenated amorphous carbon film against pairs: a physical description, J. Appl. Phys. 110 (2011) 033521.

[24] C. Donnet, A. Erdemir, Historical developments and new trends in tribological and solid lubricant coatings, Surf. Coat. Tech. 180 (2004) 76-84. 
[25] W. L. Shi, X. T. Wei, W. Zhang, Z. G. Wang, C. H. Dong, S. Li, Developments and applications of diamond-like carbon, in: Appl. Mech. Mater., volume 864, Trans Tech Publ, pp. 14-24.

[26] H. Schittenhelm, D. B. Geohegan, G. Jellison, A. A. Puretzky, M. J. Lance, P. F. Britt, Synthesis and characterization of single-wall carbon nanotube-amorphous diamond thin-film composites, Appl. Phys. Lett. 81 (2002) 2097-2099.

[27] H. Kinoshita, I. Ippei, H. Sakai, N. Ohmae, Synthesis and mechanical properties of carbon nanotube/diamond-like carbon composite films, Diam. Relat. Mater. 16 (2007) 1940-1944.

[28] A. H. Lettington, Applications of diamond-like carbon thin films, Carbon 36 (1998) 555-560.

[29] D. Varshney, B. R. Weiner, G. Morell, Growth and field emission study of a monolithic carbon nanotube/diamond composite, Carbon 48 (2010) 3353-3358.

[30] N. Sakudo, N. Ikenaga, H. Yasui, K. Awazu, Amorphous carbon coating mixed with nano-diamonds, Thin Solid Films 516 (2008) 4483-4486.

[31] J. Zhang, Y. Yu, D. Huang, Good electrical and mechanical properties induced by the multilayer graphene oxide sheets incorporated to amorphous carbon films, Solid State Sci. 12 (2010) 1183-1187.

[32] J. Baimova, L. K. Rysaeva, A. Rudskoy, Deformation behavior of diamond-like phases: Molecular dynamics simulation, Diam. Relat. Mater. 81 (2018) 154-160.

[33] Y. Wang, J. Xu, Y. Ootani, S. Bai, Y. Higuchi, N. Ozawa, K. Adachi, J. M. Martin, M. Kubo, Tight-binding quantum chemical molecular dynamics study on the friction and wear processes of diamond-like carbon coatings: Effect of tensile stress, ACS Appl. Mater. Inter. 9 (2017) 34396-34404.

[34] Y. Wang, J. Xu, J. Zhang, Q. Chen, Y. Ootani, Y. Higuchi, N. Ozawa, J. M. Martin, K. Adachi, M. Kubo, Tribochemical reactions and graphitization of diamond-like carbon against alumina give volcano-type temperature dependence of friction coefficients: A tight-binding quantum chemical molecular dynamics simulation, Carbon 133 (2018) 350-357. 
[35] W. Ren, A. Iyer, J. Koskinen, A. Kaskela, E. I. Kauppinen, K. Avchaciov, K. Nordlund, Conditions for forming composite carbon nanotubediamond like carbon material that retain the good properties of both materials, J. Appl. Phys. 118 (2015) 194306.

[36] D. McKenzie, Tetrahedral bonding in amorphous carbon, Rep. Prog. Phys. 59 (1996) 1611.

[37] J. Robertson, Diamond-like amorphous carbon, Mater. Sci. Eng. R 37 (2002) 129-281.

[38] K. Nordlund, M. Ghaly, R. Averback, M. Caturla, T. D. de La Rubia, J. Tarus, Defect production in collision cascades in elemental semiconductors and fcc metals, Phys. Rev. B 57 (1998) 7556.

[39] M. Ghaly, K. Nordlund, R. Averback, Molecular dynamics investigations of surface damage produced by kiloelectronvolt self-bombardment of solids, Philos. Mag. A 79 (1999) 795-820.

[40] D. W. Brenner, Empirical potential for hydrocarbons for use in simulating the chemical vapor deposition of diamond films, Phys. Rev. B 42 (1990) 9458.

[41] D. W. Brenner, Erratum: Empirical potential for hydrocarbons for use in simulating the chemical vapor deposition of diamond films, Phys. Rev. B 46 (1992) 1948.

[42] K. Beardmore, R. Smith, Empirical potentials for c-si-h systems with application to c60 interactions with si crystal surfaces, Philos. Mag. A 74 (1996) 1439-1466.

[43] H. Jäger, K. Albe, Molecular-dynamics simulations of steady-state growth of ion-deposited tetrahedral amorphous carbon films, J. Appl. Phys. 88 (2000) 1129-1135.

[44] N. Marks, Modelling diamond-like carbon with the environmentdependent interaction potential, Journal of Physics: Condensed Matter 14 (2002) 2901.

[45] A. Stukowski, Visualization and analysis of atomistic simulation data with ovito-the open visualization tool, Model. Simul. Mater. Sc. 18 (2009) 015012. 
[46] H. J. Berendsen, J. v. Postma, W. F. van Gunsteren, A. DiNola, J. Haak, Molecular dynamics with coupling to an external bath, J. Chem. Phys. 81 (1984) 3684-3690.

[47] X. Yuan, A. Cormack, Efficient algorithm for primitive ring statistics in topological networks, Comp. Mater. Sci. 24 (2002) 343-360.

[48] J. Liu, X. Tang, Y. Li, Z. Dai, F. Chen, H. Huang, H. Li, H. Liu, D. Chen, Effects of irradiation-induced structure evolution on the adhesion force and instantaneous modulus of multi-walled carbon nanotube arrays, Mater. Chem. Phys. 196 (2017) 160-169.

[49] J. Tersoff, Empirical interatomic potential for carbon, with applications to amorphous carbon, Phys. Rev. Lett. 61 (1988) 2879.

[50] A. A. Zavitsas, The relation between bond lengths and dissociation energies of carbon- carbon bonds, The Journal of Physical Chemistry A 107 (2003) 897-898. 